\title{
A Taxonomy of Seven-Day Readmissions to an Urban Teaching Hospital
}

\author{
Daniel Burke, MD, MBA, Nate Link, MD, MPH, Douglas Bails, MD, Yixin Fang, PhD, Michael P. Janjigian, MD*
}

Division of General Internal Medicine, Department of Medicine, New York University School of Medicine, New York, New York.

BACKGROUND: Understanding the mechanism of unplanned hospital readmissions is necessary for accurate prediction and prevention.

OBJECTIVE: To identify specific mechanisms of unplanned readmissions through medical narratives obtained from chart reviews.

DESIGN: Retrospective chart review.

SETTING: Urban tertiary care hospital.

PATIENTS: Two hundred seventy patients accounted for 335 unplanned 7-day readmissions between July 2010 and July 2011.

MEASUREMENTS: Readmissions were classified into 1 of 5 distinct categories.

RESULTS: Readmitted subjects were more likely to have had a longer length of stay during the first admission compared to nonreadmitted patients. Readmissions due to unpredictable/unpreventable complications or unrelated events constituted the highest percentage at $46 \%$.
Readmissions due to patient factors such as substance abuse, signing out against medical advice, or nonadherence to the treatment plan constituted $31 \%$. Readmissions designated as preventable accounted for $24 \%$. Among preventable readmissions, the most common cause was incomplete management of the index diagnosis. The interobserver level of agreement across the 5 major categories was substantial.

CONCLUSIONS: We found through detailed chart review of patients readmitted within 7 days to an urban teaching hospital that the majority of readmissions were not avoidable and were often due to unpredictable or unpreventable complications of the primary diagnosis from the index hospitalization or to patient behaviors that contradicted the treatment plan. These results question the value of readmissions as a valid metric of quality and support future interventions in hospital systems to reduce preventable readmissions. Journal of Hospital Medicine 2016;11:33-38. (c) 2015 Society of Hospital Medicine
Unplanned hospital readmissions are regarded as a core measure of quality of care and may comprise a large avoidable cause of healthcare expenditures. ${ }^{1-5}$ An estimated $20 \%$ of Medicare patients who are discharged from a hospital are readmitted within 30 days. ${ }^{1,6}$ This has led the Centers for Medicare \& Medicaid Services and other payers to reduce reimbursements for unplanned 30-day hospital readmissions.

Efforts to decrease readmission rates have been hampered by ineffective risk prediction models, and strategies to reduce readmissions have found limited success. ${ }^{7}$ Understanding the mechanism of readmissions is necessary for accurate prediction and prevention. This can be achieved only through analysis of patient data and medical narratives obtained from patient interviews or detailed chart reviews. ${ }^{8}$ Studies attempting to identify mechanisms of readmission

*Address for correspondence and reprint requests: Michael Janjigian, MD, Assistant Professor of Medicine, Director of Inpatient General Medicine, Bellevue Hospital, Division of General Medicine, New York University School of Medicine, New York, NY 14621; Telephone: 212-562-8360; Fax: 212-263-1048; E-mail: janjigian@gmail.com

Additional Supporting Information may be found in the online version of this article.

Received: March 18, 2015; Revised: August 11, 2015; Accepted: August 24, 2015

2015 Society of Hospital Medicine DOI 10.1002/jhm.2481

Published online in Wiley Online Library (Wileyonlinelibrary.com). using narrative chart reviews have been limited by small sample size, highly selected patient samples, and poor interobserver agreement. ${ }^{8-10}$

Our objective in this study was to identify specific mechanisms and risk factors of unplanned readmissions from the medicine service of a large urban hospital by reviewing medical charts for each case. Given the inverse relationship between time since discharge from the initial admission and the probability of an avoidable readmission, ${ }^{8}$ we focused our review on 7-day readmissions.

\section{METHODS}

\section{Setting}

The study took place within Bellevue Hospital Center, an 800-bed teaching hospital that serves a culturally and racially diverse inner-city population in New York City. Bellevue is 1 of 11 acute-care facilities managed by Health and Hospitals Corporation. The Bellevue inpatient medicine service is staffed by board-certified general internists (180 beds), oncologists (20 beds), and pulmonologists ( 20 beds), who function as hospitalists in supervision of housestaff and physicians. Their efforts are supported by case managers and social workers who meet every weekday with physicians and nurses to plan discharges as multidisciplinary teams. Weekend support is minimal, consisting of an on-call social worker to assist with 
urgent matters only. Upon discharge, patients are referred directly to 1 or more of Bellevue's outpatient clinics or to their own primary care providers outside Bellevue. There is a single electronic medical record for Bellevue, which spans the full range of care provided in the outpatient clinics, emergency department, and inpatient service.

\section{Patients}

Eligible patients were discharged from the Bellevue medical service between July 1, 2010 and July 1, 2011, and readmitted to any service at Bellevue within 7 days. During the study period, there were 8421 discharges. Discharges included transfers to other hospitals or rehabilitation centers, and excluded patients who died during hospitalization. Of these, 6781 were not readmitted, 1581 were readmitted within 30 days $(18.8 \%)$, and 549 were readmitted within 7 days $(6.5 \%)$. From the latter group, 20 consecutive cases were excluded after use in an exploratory pilot study, 84 consecutive cases were excluded after use in a formal pilot study, leaving 445 cases, from which 400 cases were randomly selected via terminal digit of the medical record number. We selected 400 chart reviews as a reasonable sample size to provide a $95 \%$ confidence interval, with a margin of error less than $4.9 \%$ for any of the proportions of the 5 readmission categories. Of these, 65 were determined to be planned readmissions (eg, for elective chemotherapy). The remaining 335 unplanned 7-day readmissions served as the subjects of this review. The study was approved by the institutional review board of New York University School of Medicine.

\section{Reviewers}

Three of the authors of this paper (Drs. Janjigian, Bails, and Link) were actively practicing boardcertified internal medicine physicians with 7, 19, and 26 years, respectively, of postresidency clinical experience during the review period of this study. Every case was reviewed by 2 investigators. One author (Drs. Janjigian) reviewed readmissions from the first 6 months of the calendar year, the second (Dr. Bails) reviewed readmissions from the last 6 months, and the third (Dr. Link) reviewed all 335 readmissions.

\section{Data Collection}

Using the electronic medical record, each readmission was reviewed with the intent to identify the sequence of events leading up to the readmission, most commonly achieved by analyzing the discharge summary from the initial admission and the admission note from the second admission. Further chart review was completed as necessary to establish the clearest narrative and to classify the readmission into 1 of 5 categories based on the cause. Narratives are defined here as the sequence of events leading to the readmission as determined by chart review and not by patient interviews. Narratives were recorded for each case to assist with understanding how each author determined the classification, and were used when disagreements required group consensus. Time spent on individual chart reviews varied widely, from 1 to 30 minutes, depending on the complexity of each case. For example, an against medical advice (AMA) discharge could be immediately identified in the medical record, whereas a determination that an incomplete workup was conducted would require reviewing the admission note from the readmission, the discharge summary from the index admission, review of progress and consult notes, and even vital signs, labs, and radiology.

An algorithm for classifying contributory causes of readmission into 1 of 5 categories was created from narratives compiled from a pilot of 84,7 -day readmissions to Bellevue during the previous year. Six readmitted patients were interviewed by a study author during this pilot phase. These narratives were determined by consensus of the authors to provide no additional relevant information from that obtained through chart review alone. The 5 categories are identified in Figure 1 as follows:

1. Second admission was not medically necessary.

2. Second admission followed an elopement (patient left without knowledge of the hospital staff) or discharge AMA during the first admission.

3. Second admission was caused by a deficiency in the discharge process of the first admission, attributable to the hospital system or providers.

4. Second admission was caused by a factor attributable to the patient including substance use or nonadherence to the treatment plan from the first admission.

5. Second admission was related to a complication of the primary disease or its treatment or an unrelated condition that could not reasonably have been predicted or prevented by a competent physician meeting the standard of care.

Categories 3, 4, and 5 were further divided into more specific subcategories as shown in Figure 1.

Each readmission was assigned a single category from the algorithm using a stepwise process in which a higher-order cause excluded consideration of a downstream category. For example, if the second admission was not medically necessary (category 1 ), an incorrect decision to readmit the patient was considered the primary cause of the readmission, and no consideration was given to categories 2 through 5 . In this manner, each patient was assigned to a single category. We considered readmissions attributable to provider error (categories 1 and 3) to be avoidable. Examples of readmissions in each category with narratives are shown in the Supporting Information, Appendix 1, in the online version of this article. Discrepancies in classification were resolved by consensus of all authors.

\section{Statistical Analysis}

Unweighted kappa values were measured to assess agreement between authors in the assignment of the major 
1. Was the readmission medically necessary?

a. Based on information available at the time of the readmission, could the patient have been safely treated as an outpatient? (Assuming 48 hour availability of an outpatient appointment and reasonable availability of standard outpatient services.)

b. If $\mathrm{NO}$ - proceed to question 2.

2. Was the initial discharge against medical advice?

a. Did the patient elope or sign out against medical advice during the first hospitalization?

b. If $\mathrm{NO}$ - proceed to question 3

3. Was the readmission related to a deficiency in the initial discharge process? If YES, select the most suitable category below. If NO, proceed to step 4 .

a. Prescription of medication - was the need for readmission related to the selection or dosing of one or more medications prescribed at the initial discharge? If YES, select the most suitable category below.

i. Overdosing of a prescribed medication

ii. Underdosing of a prescribed medication

iii. Unanticipated adverse effect of a prescribed medication

iv. Interaction of two or more prescribed medications

v. Other - specify

b. Timing of initial discharge - was the readmission related to prematurity of the initial discharge? If YES, select the most suitable category below.

i. Patient's functional status inadequate at time of discharge (e.g., discharged to home but not able to ambulate)

ii. Clinical management (diagnosis or treatment) incomplete at time of discharge

c. Discharge plan - was the readmission related to inadequacy in the planning or execution of the discharge process? If YES, select the most suitable category below.

i. Patient unable to fill prescriptions (e.g., prescription not matched to insurance-specific formulary)

ii. Outpatient followup arrangements not adequate for patient's needs

iii. Discharge setting (e.g., home, hospice, or chronic care facility) not appropriate for patient's needs

iv. Inadequate communication of discharge plan to all appropriate parties (e.g., nursing home not told of need for contact isolation)

v. Other-specify

4. Was the readmission related to patient behavior?

a. Was the readmission related to substance use (e.g. alcohol withdrawal)?

b. Was the readmission otherwise related to poor patient adherence to the treatment and discharge plan?

c. Was the readmission related to patient/family refusal to comply with recommended discharge plan?

5. Was the readmission related to another reason not specified above?

a. Was the readmission caused by an unpredictable or unpreventable event related to the primary diagnosis or its treatment (e.g. atrial fibrillation led to a stroke despite adequate anticoagulation, pacemaker malfunctioned)?

b. Was the readmission caused by an unpredictable event related to a new diagnosis (e.g. trauma)

c. Other - specify

FIG. 1. Algorithm for Categorizing Primary Cause of Readmission.

category among the 5 choices in the algorithm. $\chi^{2}$ tests were used to compare categorical variables between 2 groups (readmitted vs not readmitted) or between several groups (5 categories of readmissions), whereas KruskalWallis tests were used for continuous variables.

Only the first readmission was used in analysis of patient characteristics when multiple readmissions occurred for an individual patient. Unique patients were used for analysis of nonreadmitted patients. The generalized estimating equation method was used to adjust for correlations between multiple readmissions within patients.

\section{RESULTS}

During this period, 270 patients accounted for 335 readmissions. Characteristics of patients readmitted within 7 days are shown in Table 1 and compared with those of patients who were not readmitted during the same study period. Patients who were readmitted were more likely to have had a longer length of stay during the first admission.

Results of categorization of readmission are shown in Table 2. Readmissions related to the discharge process (category 3) were further divided into subcategories (Table 3). Category 5 (unpredictable/unpreventable complication of primary diagnosis or unrelated event) constituted the highest percentage of readmissions at $46 \%$, followed by category 4 (patient behavior) at 19\%, category 3 (discharge process deficiency) at 17\%, category 2 (AMA) at $12 \%$, and category 1 (unnecessary admission) at $7 \%$. Readmissions designated as preventable (categories 1 and 3) accounted for $24 \%$ of all readmissions. 
TABLE 1. Patient Characteristics

\begin{tabular}{lccc}
\hline Characteristic & $\begin{array}{c}\text { Not Readmitted, } \\
\mathrm{n}=6,781\end{array}$ & $\begin{array}{c}\text { Readmitted, } \\
\mathrm{n}=270\end{array}$ & $P$ Value \\
\hline Male gender (\% of category) & $4,224(62.3 \%)$ & $180(66.7 \%)$ & 0.15 \\
Mean age, y (SD) & $56.1(16.3)$ & $55.1(16.3)$ & 0.65 \\
Median initial LOS [interquartile range] & $3[2,6]$ & $4[2,9]$ & 0.002 \\
Mean days between admissions (SD) & $\mathrm{NA}$ & $3.8(2.1)$ & $\mathrm{NA}$ \\
AMA discharge (\% of category) & $413(6.1 \%)$ & $20(7.4 \%)$ & 0.38 \\
\hline
\end{tabular}

NOTE: Abbreviations: LOS, length of stay; NA, not applicable; SD, standard deviation.

Readmissions due to patient factors (categories 2 and 4) accounted for $31 \%$ of all readmissions. Notably, $21 \%$ of all readmissions were due to patients who eloped or left AMA during the first discharge or who returned because of substance abuse during the interim (categories 2 and $4 a)$. Among the preventable readmissions, the most commonly designated cause of readmission was a perceived premature discharge (category $3 \mathrm{~b} 2$ ), accounting for $6 \%$ of all readmissions.

Variance was statistically significant across major categories for gender, mean age, and median length of stay. The interobserver level of agreement across the 5 major categories was substantial among both pairs of reviewers (Table 4).

The 46 patients who had more than 1, 7-day readmission during this study period were responsible for 106 readmissions. The majority of this group were readmitted twice $(78 \%)$, with a range of 2 to 5 readmissions. Within this group, $24 \%$ were considered preventable readmissions ( 8 from category 1,17 from category 3 ), and $76 \%$ were considered not preventable (10 from category 2, 27 from category 4, and 44 from category 5).

\section{DISCUSSION}

The purpose of this retrospective review was to identify causes of unplanned 7-day readmissions after discharge from the medical service of a large urban teaching hospital. Rather than focus on risk factors for readmissions, which other studies have done, we reviewed charts of readmitted patients using a novel categorization algorithm to group patients into common mechanisms that elucidate why a particular patient was
TABLE 3. Characteristics of Categories 3, 4, and 5

\begin{tabular}{llrc}
\hline Category & \multicolumn{1}{c}{ Description } & No. & \% of Total \\
\hline 3a1 & Overdosing of a prescribed medication & 3 & 0.9 \\
3a2 & Underdosing of a prescribed medication & 5 & 1.5 \\
$3 \mathrm{a} 3$ & Adverse medication effect & 2 & 0.6 \\
$3 \mathrm{~b} 1$ & Inadequate functional status & 3 & 0.9 \\
3b2 & Premature discharge & 20 & 6.0 \\
3c1 & Patient unable to fill prescriptions & 9 & 2.7 \\
3c2 & Follow-up arrangements inadequate & 6 & 1.8 \\
3c3 & Discharge setting not appropriate & 5 & 1.5 \\
3c4 & Inadequate communication of plan to receiving facility & 2 & 0.6 \\
3c5 & Other & 1 & 0.3 \\
$4 \mathrm{a}$ & Patient behavior-substance use & 30 & 9 \\
4b & Patient behavior-adherence to discharge plan & 30 & 9 \\
$4 \mathrm{c}$ & Patient behavior-refusal of discharge plan & 3 & 0.9 \\
5a & Disease complication & 103 & 30.7 \\
$5 \mathrm{~b}$ & Unrelated condition & 52 & 15.5 \\
\hline
\end{tabular}

readmitted. By examining the chart in detail, we were able to identify etiologies of readmission that are potentially avoidable.

Some authors have questioned the use of readmissions as a measurement of the quality of care a hospital provides due to the high proportion of unavoidable readmissions in a given sample. ${ }^{8,10}$ We hoped to identify systems errors that could be targets of quality improvement initiatives, and therefore chose to focus entirely on 7-day readmissions as these have been shown to be more preventable than 30 -day readmissions. ${ }^{8}$ We had the ability to review any aspect of the medical chart (eg, vitals or labs on discharge, any clinical note), which provided the highest probability of discovering a systems error. Despite these efforts to identify preventable errors, we identified the most common mechanism of readmission as an unpredictable or unpreventable event related to the primary diagnosis or its treatment from the initial admission (category $5 \mathrm{a}, 30.7 \%$ of total readmissions). Review of examples from this category elucidates how an unpredictable readmission could occur within such a short time frame (see Supporting Information, Appendix 1, in the online version of this article). The 7-day window precluded identification of clinic access barriers, thereby eliminating from analysis 1 mechanism for preventable readmissions.

\section{TABLE 2. Readmission Characteristics Based on 335 Readmissions}

\begin{tabular}{|c|c|c|c|c|c|c|}
\hline & $\begin{array}{l}\text { Category } 1 \text { : Second } \\
\text { Admission Not } \\
\text { Medically Necessary }\end{array}$ & $\begin{array}{l}\text { Category 2: First } \\
\text { Admission AMA }\end{array}$ & $\begin{array}{c}\text { Category } 3: \\
\text { Deficiency in the } \\
\text { Discharge Process }\end{array}$ & $\begin{array}{c}\text { Category 4: } \\
\text { Patient Behavior }\end{array}$ & $\begin{array}{c}\text { Category } 5: \\
\text { Unpredictable Complication } \\
\text { of Primary or } \\
\text { Alternate Diagnosis }\end{array}$ & $P$ Value $^{*}$ \\
\hline Total (\%) & $22(6.6 \%)$ & $39(11.6 \%)$ & $56(16.7 \%)$ & $63(18.8 \%)$ & $155(46.3 \%)$ & \\
\hline Male (\%) & $11(50.0 \%)$ & $29(74.4 \%)$ & $38(67.9 \%)$ & $54(85.7 \%)$ & $91(58.7 \%)$ & 0.005 \\
\hline Mean age, y (SD) & $61.8(13.7)$ & $48.1(13.2)$ & $58.6(14.4)$ & $53.3(11.8)$ & $55.1(17.7)$ & 0.004 \\
\hline Median LOS [IQR] & $2.5[2.0,7.0]$ & $2.0[1.0,6.0]$ & $5.0[2.0,8.5]$ & $4.0[2.0,6.0]$ & $5.0[2.0,10.0]$ & 0.03 \\
\hline $\begin{array}{l}\text { Mean days between } \\
\text { admissions (SD) }\end{array}$ & $3.8(2.2)$ & $3.1(2.2)$ & $3.3(2.0)$ & $3.8(2.1)$ & $4.1(2.1)$ & 0.27 \\
\hline
\end{tabular}


TABLE 4. Disagreements Between Reviewers

\begin{tabular}{lccc}
\hline Pair of Reviewers & $\begin{array}{c}\text { No. of Readmissions } \\
\text { Reviewed }\end{array}$ & $\begin{array}{c}\text { No. of } \\
\text { Agreements (\%) }\end{array}$ & $\begin{array}{c}\text { Unweighted } \\
\text { Kappa }\end{array}$ \\
\hline Dr. Link-Dr. Bails & 135 & $113(83.7)$ & 0.78 \\
Dr. Link-Dr. Janjigian & 200 & $163(81.5)$ & 0.72 \\
\hline
\end{tabular}

Nonetheless, our study demonstrates room for improvement in provider behavior and hospital systems related to the discharge process. Nearly a quarter of all readmissions and the majority of preventable readmissions were related to systems issues, such as timing and coordination of the first discharge, and lack of medical necessity for the second admission (see Supporting Information, Appendix 1, in the online version of this article). Prior studies found that shorter length of stay was associated with increased preventable readmissions, a finding that our study does not support. ${ }^{10,11}$ We suspect that patients in this group had longer lengths of stay during the index hospitalization due to complexity of medical illness, limited social support network, or lack of insurance, among other factors, that exposed flaws in systems processes and provider judgment. The mechanisms of readmission related to discharge planning that we identified in this study, including comprehensiveness of care, coordination of care, and medication administration, all represent potential opportunities for intervention.

Of note, there was a high percentage of readmissions attributable to patient behaviors, such as AMA discharges, substance abuse following discharge, and nonadherence to the treatment plan. These factors are likely over-represented in the Bellevue patient population compared to that of private hospital settings and no doubt exacerbate the readmission rates in urban hospitals treating patients with a high degree of social and behavioral health needs. Although patient-related factors such as AMA discharges and substance abuse are potentially addressable, our reviewers felt that these were not preventable based on current knowledge and standards of care.

Studies that have attempted to classify readmissions as potentially avoidable have not shown good interobserver agreement when more than 1 reviewer was involved. ${ }^{9,10}$ Additionally, there is not a validated tool available to classify types of readmissions. By using a pilot sample of 84 cases to develop the model, confirming the accuracy of the chart by personally interviewing a sample of readmitted patients for comparison, and by employing experienced inpatient attending physicians to perform the reviews, we were able to develop an algorithm that achieved substantial reliability in assigning each readmission into 1 of 5 distinct categories.

Our literature search revealed only a single study that attempted to classify readmissions in a similar manner. Readmissions within 6 months at 9 Veterans Affairs hospitals were classified into causal categories of systems, provider, and patient etiology. ${ }^{9}$ Overall,
$34 \%$ of readmissions were deemed to be preventable compared to $24 \%$ in our study. Most readmissions $(68 \%)$ were due to a worsening of a clinical condition, $4.5 \%$ were attributed to the admitting provider having too low a threshold to justify admission, and $2.7 \%$ were due to the patient not abstaining from drugs or alcohol. Though the study design and patient population differed from our own, the similarities in methods and results lend validity to the results and conclusions of our study.

Another limitation of our study is that readmissions to other hospitals were not included. In this respect, our estimate of the rate of readmission was an understatement of the true value. Nonetheless, the categorization of causes for readmission was not likely to be affected by the site of the second admission. Another limitation of this study was the small number of subjects reviewed relative to other studies that analyzed demographics and risk factors in large databases of readmissions. ${ }^{12}$ However, the depth of the present review provides an understanding of the sequence of events leading to the readmission and permits development of strategies to prevent their occurrence.

We identified mechanisms of readmissions that can lay the groundwork for future interventions and safely reduce readmissions rates at little cost. To reduce admissions that may not be medically necessary, the narratives presented in the supplementary appendix suggest that improvement in communication between the admitting provider for the readmission and a provider familiar with the patient could have led to avoidance of the readmission. Similarly, enhanced communication to receiving nursing facilities would decrease the chances of the patient being immediately sent back as occurred multiple times in our cohort. Formal mandatory assessment of functional status for vulnerable patients would identify patients who may not be fully ready for discharge.

In conclusion, we found through detailed chart review of patients readmitted within 7 days to an urban teaching hospital that the majority of readmissions were not avoidable and were due to unpredictable complications of the primary diagnosis from the index hospitalization or a condition unrelated to the initial stay. This conclusion, in concurrence with those of other studies, ${ }^{8,10}$ questions the value of a readmission as a valid metric of quality though supports further improvements in hospital systems to reduce preventable readmissions.

Disclosure: Nothing to report.

\section{References}

1. Jencks SF, Williams MV, Coleman EA. Rehospitalizations among patients in the Medicare fee-for-service program. N Engl J Med. 2009; 360(14):1418-1428.

2. Friedman B, Basu J. The rate and cost of hospital readmissions for preventable conditions. Med Care Res Rev. 2004;61(2):225-240.

3. Centers for Medicare and Medicaid Services. 9th scope of work version \#080108-0. Available at: http://www.cms.gov/Medicare/Quality-InitiativesPatient-Assessment-Instruments/QualityImprovementOrgs/downloads/ 9thSOWBaseContract_C_08-01-2008_2_.pdf. Accessed April 10, 2014. 
4. Anderson GF, Steinberg EP. Hospital readmissions in the Medicare population. N Engl J Med. 1984;311(21):1349-1353.

5. U.S. Department of Health \& Human Services. Hospital Compare. Available at: http://www.hospitalcompare.hhs.gov. Accessed April 12, 2014.

6. Medicare Payment Advisory Commission. Report to the congress: reforming the delivery system. June 2008. Available at: http://www.medpac.gov/documents/reports/Jun08_EntireReport.pdf?sfvrsn=0. Accessed September 15, 2015.

7. Hansen LO, Young RS, Hinami K, Leung A, Williams MV. Interventions to reduce 30-day rehospitalization: a systematic review. Ann Intern Med. 2011;155(8):520-528.

8. van Walraven C, Jennings A, Taljaard M, et al. Incidence of potentially avoidable urgent readmissions and their relation to all-cause urgent readmissions. CMAJ. 2011;183(14):E1067-E1072.
9. Oddone EZ, Weinberger M, Horner M, et al. Classifying general medicine readmissions. Are they preventable? Veterans Affairs Cooperative Studies in Health Services Group on Primary Care and Hospital Readmissions. J Gen Intern Med. 1996;11(10):597-607.

10. Koekkoek D, Bayley KB, Brown A, Rustvold DL. Hospitalists assess the causes of early hospital readmissions. J Hosp Med. 2011;6(7): 383-388.

11. Balla U, Malnick S, Schattner A. Early readmissions to the department of medicine as a screening tool for monitoring quality of care problems. Medicine. 2008;87(5):294-300.

12. Hanlon JT, Pieper CF, Hajjar ER, et al. Incidence and predictors of all and preventable adverse drug reactions in frail elderly persons after hospital stay. J Gerontol A Biol Sci Med Sci. 2006;61(5):511515. 